\title{
Measuring starspot temperature from line depth ratios ${ }^{\star}$
}

\section{The method}

\author{
S. Catalano ${ }^{1}$, K. Biazzo ${ }^{2}$, A. Frasca ${ }^{1}$, and E. Marilli ${ }^{1}$ \\ ${ }^{1}$ Catania Astrophysical Observatory, via S. Sofia 78, 95123 Catania, Italy \\ 2 Dep. of Physics and Astronomy, University of Catania, via S. Sofia 78, 95123 Catania, Italy
}

Received 8 April 2002 / Accepted 29 July 2002

\begin{abstract}
Gray and collaborators have recently demonstrated that line-depth ratios are a powerful tool for temperature discrimination, able to resolve differences $\leq 10 \mathrm{~K}$.

The method has been applied to detect temperature variations in the 5-15 K range due to activity cycles (e.g. Gray et al. 1996a, 1996b) or to rotation modulation produced by large surface features, called "star-patches", like that detected in $\xi$ Boo A by Toner \& Gray (1988).

Cool starspots of a few tenths of the stellar surface produce bumps in a line profile, which migrate through the line profile allowing Doppler-imaging in fast rotating stars. In the hypothesis that in slowly-rotating stars the passage of dark spots produces modulation of the center line depth of different amount in lines of different sensitivity to temperature, we have made test observations on three active binaries of the RS CVn type.

Based on observations made at the Catania Astrophysical Observatory at a resolution $R=14000$, we show that line-depth ratios can be effectively used to determine spot temperatures of active binary systems.

Using, on average, ten line pairs, selected in the 6100-6300 $\AA$ wavelength range, with the help of observations of 30 main sequence and giant stars, we have derived a calibration relation of line-depth ratios (LDR) in an absolute temperature scale, taking into account the gravity effect in the calibration relation.

Single LDRs converted to temperature through the calibration relations have led to clear rotational modulation of the average surface temperature with amplitudes of $177 \mathrm{~K}, 119 \mathrm{~K}$, and $127 \mathrm{~K}$ for VY Ari, IM Peg and HK Lac, with average estimated errors of about $10 \mathrm{~K}$.

We show that the observed temperature variation amplitude allows us to define a minimum fractional spotted area coverage as a function of spot-photosphere temperature ratio.

Adopting the maximum value of average temperature, determined from the LDRs, as that of the unspotted photosphere, we computed the average spot temperature corresponding to the minimum spot coverage. Although not univocally constrained, the temperature difference ( $\Delta T=T_{\mathrm{ph}}-T_{\mathrm{sp}}$ ) obtained for the three systems, $\Delta T=890 \mathrm{~K}$ for VY Ari, $\Delta T=750 \mathrm{~K}$ for IM Peg, and $\Delta T=810 \mathrm{~K}$ for HK Lac, are in good agreement with values derived with other methods.
\end{abstract}

Key words. stars: late-type - stars: activity - stars: starspots - stars: individual: VY Ari, HK Lac, IM Peg

\section{Introduction}

Photometric variations attributed to starspots clearly depend on a number of physical parameters, i.e. size, location, effective temperature of the spots or spot groups. The numerous attempts made to extract this information by modelling the photometric light curve unavoidably meet with the non-uniqueness of the solutions. While a certain spot configuration can reasonably reproduce the photometric variations, it is not possible to invert models to derive unique values for spot sizes, shapes and temperatures. There is a fundamental trade-off between spot area

Send offprint requests to: S. Catalano,

e-mail: scatalano@ct.astro.it

* Based on observations collected at Catania Astrophysical Observatory, Italy. and spot temperature. Very precise data at least in two colors are required to resolve this ambiguity. Unfortunately the most used color index, the $B-V$, is not of great help because, as the spot becomes darker its contribution to the color of the visible stellar hemisphere decreases and the observed color variation associated with the rotational modulation is always small. The use of the $V-R$ color is more effective for the contemporaneous determination of starspot temperature and area, as earlier shown by Vogt (1981). The method determines the $V-R$ color difference between the star and spot, while the spot temperature is derived through the $(V-R)-T_{\text {eff }}$ calibration.

The Doppler-imaging technique (e.g. Vogt et al. 1987; Piskunov 1991) based on a series of high resolution spectral line profiles allows us to produce an image of the stellar surface with high degree of sophistication and accuracy (for recent 
developments see Rice \& Strassmeier 2000). However, the temperature scale remains model dependent, in the sense that with a given set of stellar atmosphere models, the stellar image can be considered as the distribution of effective temperature across the stellar surface. The temperature scale of the image can be fixed by simultaneous photometric observations.

Following the earlier suggestions that $\mathrm{TiO}$ bands at $8860 \AA$ could be used to measure starspot temperatures (e.g. Vogt 1979; Ramsey \& Nations 1980), Huenemoerder \& Ramsey (1987) attempted a quantitative study of the effect of spots on the $\mathrm{TiO}$ bands to derive spot temperatures and surface coverage. Recently Neff et al. (1995) and O'Neal et al. (1996) obtained an evaluation of spot temperature with an estimated error of $\pm 100-200 \mathrm{~K}$ and of the surface filling factor for a number of active stars using both bands of TiO at 7055 and $8860 \AA$. Although the method looks very effective for stars with spot temperatures lower than $3500 \mathrm{~K}$, it fails for stars with effective temperature higher than $5000 \mathrm{~K}$ if the average temperature difference is $T_{\text {eff }}-T_{\mathrm{sp}} \leq 1500 \mathrm{~K}$, as generally observed. Moreover, the method requires an independent determination of $T_{\text {eff }}$.

In a series of recent works Gray and collaborators have demonstrated that line-depth ratios are a powerful temperature discriminant, capable of resolving differences $\leq 10 \mathrm{~K}$, and have determined $T_{\text {eff }}$ of several main sequence (Gray \& Johanson 1991) and giant stars (Gray \& Brown 2001). Although the effective temperature scale calibration can be set to few tens of a degree, temperature differences can be measured with a precision less than one degree. Temperature variation in the 5-15 K range over the rotational period and the activity cycle has been reported for $\epsilon$ Eri (Gray \& Baliunas 1995), $\sigma$ Dra (Gray et al. 1992), as well as for the Sun along the 11-year cycle (Gray \& Livingston 1997).

Application of this method to the study of temperature variations associated with stellar surface features, as discussed by Gray (1996), is particularly challenging because several physical variables interact simultaneously, each impressing their signature on the spectral lines. In particular, cool surface features (spots) cause bumps in a line profile, which migrate through the line profile allowing Doppler-imaging in fast rotating stars. Since the size of the bump, to the first order, is determined by the loss of continuum light at the Doppler position of the bump, in a slowly rotating star we cannot see a clear Doppler shift but can expect a slight rotational modulation of the central line depth. For example, following Gray (1996), if the passage of a dark spot at the central meridian produces a decrease of $10 \%$ in the stellar disk light, then the bump would have a height of $10 \%$ of the depth of the line. The line depth will be reduced by about this quantity.

Let us now consider the line-depth ratios in a slowly rotating star and select two lines, one insensitive and one very sensitive to temperature. Due to the presence of a dark spot that produces a decrease of $10 \%$ in the continuum, the depth of both lines would be equally affected, and reduced by $10 \%$ and their depth ratio would remain unchanged. But due to the lower temperature in the spot, the line sensitive to the temperature will change its intrinsic depth and consequently also the depth ratio of the two lines will change. The amount of depth ratio variation should depend on the sensitivity to the temperature variation of the specific lines considered, and the fraction of surface covered by the spot.

On the basis of these considerations we have made some test observations on rather active, slowly rotating RS CVn binaries, namely VY Ari, IM Peg and HK Lac to investigate the applicability of the method and its ability to determine the spot temperature. The paper develops as follows: in Sect. 2 we present the observations and data reduction; in Sect. 3 the temperature calibration of the line-depth ratios; in Sect. 4 the results of temperature modulation of the active stars will be discussed, and in Sect. 5 a method to determine the spot temperature and the filling factor will be discussed.

\section{Data analysis}

\subsection{Observations and reduction}

Spectroscopic observations have been performed with the REOSC échelle spectrograph at the 91-cm telescope of Catania Astrophysical Observatory - M. G. Fracastoro station (Serra La Nave, Mt. Etna). The spectrograph is fed by the telescope through an optical fibre (UV - NIR, $200 \mu \mathrm{m}$ core diameter) and is placed in a stable position in the room below the dome level. Spectra were recorded on a CCD camera equipped with a thinned back-illuminated SITe CCD of $1024 \times 1024$ pixels (size $24 \times 24 \mu \mathrm{m}$ ). The échelle crossed configuration yields a resolution of about 14000 , as deduced from the FWHM of the lines of the Th-Ar calibration lamp. The observations have been made in the red region. The detector allows us to record five orders in each frame, spanning from about 5860 to $6700 \AA$. In this spectral region there are several line pairs of low and high excitation potential, whose depth ratios are suitable for effective temperature determination.

The data reduction was performed by using the ECHELLE task of IRAF $^{1}$ package following the standard steps: background subtraction, division by a flat field spectrum given by a halogen lamp, wavelength calibration using the emission lines of a ThAr lamp, and normalization to the continuum through a polynomial fit. Particular care was paid to the continuum level definition. The IRAF task conTINUUM was used for such a purpose and we chose a low-order (3rd-4th) Legendre polynomial to follow the continuum behaviour in each spectral order that is the result of the true spectral shape and of residual instrumental effects, like the blazing curvature, spectrograph sensitivity, etc., that are not fully removed by the reduction process.

The choice of a low-order polynomial guarantees a good definition of the continuum level at least on a spatial scale of a few tens of $\AA$, much more than the typical wavelength separation of line pairs.

Observations were carried out from August 2000 to January 2001, on three selected targets, i.e. VY Ari, HK Lac, IM Peg, whose main parameters are reported in Table 1. The main requirement for the selection was the known strong spottedness and low $v \sin i$. The $v \sin i$ of the targets is in

\footnotetext{
${ }^{1}$ IRAF is distributed by the National Optical Astronomy Observatory, which is operated by the Association of the Universities for Research in Astronomy, inc. (AURA) under cooperative agreement with the National Science Foundation.
} 
Table 1. Main parameters of observed active stars.

\begin{tabular}{rccccr}
\hline \hline HD & Name & Sp. Type & $\begin{array}{c}V \\
(\mathrm{mag})\end{array}$ & $\begin{array}{r}v \sin i \\
\left(\mathrm{~km} \mathrm{~s}^{-1}\right)\end{array}$ & $\begin{array}{r}P_{\text {rot }} \\
(\text { days })\end{array}$ \\
\hline 209813 & HK Lac & K0III & 6.52 & 15 & 24.4284 \\
216489 & IM Peg & K2III-II & 5.60 & 26 & 24.494 \\
17433 & VY Ari & K3-4V-IV & 6.9 & 6 & 16.1996 \\
\hline
\end{tabular}

the 6-24 $\mathrm{km} \mathrm{s}^{-1}$ range, so if the spots or the spotted area is large enough to feel the rotation broadening effect the width of the bumps caused by the spot visibility would be of the same order as the width of a spectral resolution element. The slow rotation combined with the relatively low spectral resolution will ensure the non-detection of the Doppler shift of the bump along the spectral lines due to the spot rotation.

In addition to the active stars, a number of giant and main sequence stars of spectral type in the range G2III-M0III and F8V-K7V, respectively, have been observed to establish the temperature scale of the line-depth ratios.

The average signal-to-noise ratio $(S / N)$ at continuum in the spectral region of interest was $200-500$ for the calibration stars and about 100-150 for the active stars.

\subsection{Calibration stars and temperature scale}

To convert the depth ratio variation of our active stars into temperature variation we need to define a temperature scale for the measured line-depth ratios. We have then observed a number of single stars of different spectral type in the range from F8 to M0 and luminosity class from V to III. Main criteria for the selection were: (i) a low rotation velocity, (ii) a reasonably good parallax value, (iii) accurate $B-V$ color index. Since the linedepth ratio is dependent on gravity also for some temperaturesensitive lines, we have observed main sequence and giant stars to correct the gravity effect and eventually set separate temperature scales to be used for active main sequence and giant stars. The calibration stars are listed in Table 2 together with their spectral type, $V$ magnitude, parallax, $B-V$ and effective temperature. Spectral types are from the Bright Star Catalogue (Hoffleit \& Warren 1991), visual magnitudes $V$ and $B-V$ color indices are from the Geneva Web database (Mermilliod et al. 1997) and the parallaxes are from the Hipparcos Catalogue (ESA 1997).

Since effective temperatures are available for very few of our calibration stars, we used color indices $B-V$ to set the effective temperature of each calibration star. Although interstellar reddening is not expected to be large, since all the stars in Table 2, with few exceptions, are closer than 100 pc, we applied an isotropic extinction correction to obtain the $(B-V)_{0}$. We used $A_{\mathrm{V}}=0.8 \mathrm{mag} \mathrm{kpc}^{-1}$ and a ratio of total to selective extinction of 3.3 as suggested by Henry et al. (2000). Conversion of $(B-V)_{0}$ to effective temperature has been made through the empirical relation proposed by Gray (1992):

$$
\begin{aligned}
\log T_{\mathrm{eff}}= & 3.988-0.881(B-V)_{0}+2.142(B-V)_{0}^{2} \\
& -3.614(B-V)_{0}^{3}+3.2637(B-V)_{0}^{4} \\
& -1.4727(B-V)_{0}^{5}+0.2600(B-V)_{0}^{6} .
\end{aligned}
$$

Table 2. Standard stars observed for the depth- $T_{\text {eff }}$ calibrations.

\begin{tabular}{rcccrcc}
\hline \hline HD & Name & Sp. T. & $\begin{array}{c}V \\
(\mathrm{mag})\end{array}$ & $\begin{array}{c}\pi \\
(\mathrm{mas})\end{array}$ & $B-V$ & $\begin{array}{r}T_{\text {eff }} \\
(\mathrm{K})\end{array}$ \\
\hline \multicolumn{7}{c}{ GIANTS } \\
161239 & $84 \mathrm{Her}$ & G2IIIb & 5.714 & 26.13 & 0.654 & 5732 \\
196755 & $\kappa$ Del & G2IV & 5.069 & 33.27 & 0.705 & 5583 \\
161797 & $\mu$ Her & G5IV & 3.417 & 119.05 & 0.752 & 5451 \\
188512 & $\beta$ Aql & G8IV & 3.715 & 72.95 & 0.855 & 5183 \\
23249 & $\delta$ Eri & K0IV & 3.527 & 110.58 & 0.922 & 5024 \\
62345 & $\kappa$ Gem & G8IIIa & 3.568 & 22.73 & 0.932 & 5001 \\
216131 & $\mu$ Peg & G8III & 3.488 & 27.95 & 0.934 & 4996 \\
22796 & 12 Tau & G6III & 5.565 & 8.14 & 0.934 & 4996 \\
28100 & $\pi$ Tau & G7IIIa & 4.692 & 7.17 & 0.982 & 4891 \\
197989 & $\epsilon$ Cyg & K0III & 2.467 & 45.26 & 1.034 & 4783 \\
74442 & $\delta$ Cnc & K0IIIb & 3.937 & 23.97 & 1.082 & 4687 \\
12929 & $\alpha$ Ari & K2III & 2.009 & 49.48 & 1.153 & 4552 \\
54719 & $\tau$ Gem & K2III & 4.407 & 10.81 & 1.261 & 4350 \\
43232 & $\gamma$ Mon & K1.5III & 3.972 & 5.06 & 1.320 & 4239 \\
49161 & 17 Mon & K4III & 4.758 & 6.73 & 1.394 & 4096 \\
69267 & $\beta$ Cnc & K4III & 3.532 & 11.23 & 1.481 & 3923 \\
29139 & $\alpha$ Tau & K5III & 0.868 & 50.09 & 1.537 & 3813 \\
60522 & $v$ Gem & M0IIIb & 4.058 & 13.57 & 1.539 & 3809 \\
\hline & & DWARFS & & & \\
187691 & 54 Aql & F8V & 5.116 & 51.57 & 0.552 & 6045 \\
22484 & 10 Tau & F9IV-V & 4.290 & 72.89 & 0.574 & 5976 \\
157214 & 72 Her & G0V & 5.394 & 69.48 & 0.619 & 5837 \\
186408 & 16 CygA & G1.5Vb & 5.960 & 46.25 & 0.645 & 5758 \\
217014 & 51 Peg & G2.5IV & 5.463 & 65.10 & 0.665 & 5699 \\
20630 & $\kappa 1$ Cet & G5V & 4.836 & 109.18 & 0.679 & 5658 \\
10700 & $\tau$ Cet & G8V & 3.496 & 274.17 & 0.727 & 5520 \\
3651 & 54 Psc & K0V & 5.879 & 90.03 & 0.849 & 5197 \\
22049 & $\epsilon$ Eri & K2V & 3.726 & 310.75 & 0.882 & 5117 \\
16160 & HR 753 & K3V & 5.821 & 138.72 & 0.972 & 4912 \\
201091 & 61 CygA & K5V & 5.224 & 287.13 & 1.169 & 4522 \\
201092 & 61 CygB & K7V & 6.046 & 285.42 & 1.360 & 4162 \\
\hline & & & & & &
\end{tabular}

This relation is quite accurate for $(B-V)_{0} \leq 1.5$, i.e. within the color range of our calibration stars.

The metallicity effects can alter the $B-V$ indices. Gray (1994) has investigated the influence of metallicity on color indices, finding an empirical relation between $B-V$ and $[\mathrm{Fe} / \mathrm{H}]$. The $B-V$ color index is only very slightly dependent on $[\mathrm{Fe} / \mathrm{H}]$, its maximum variation being of about 0.015 . According to the calibration relation given in Eq. (1), the corresponding temperature change is about $20-30 \mathrm{~K}$. Given the uncertainties in the $B-V$ values and in the setting of the temperature scale, such effects appears to be statistically not significant in our LDR-temperature calibrations.

\subsection{Line identification in the $6100-6300 \AA$ range}

Within the spectral range covered by our échelle frames, 5870-6700 $\AA$, there are several pairs of lines suitable for temperature determination, the more frequently used being in the spectral region around $6200 \AA$ (Gray \& Johanson 1991; 


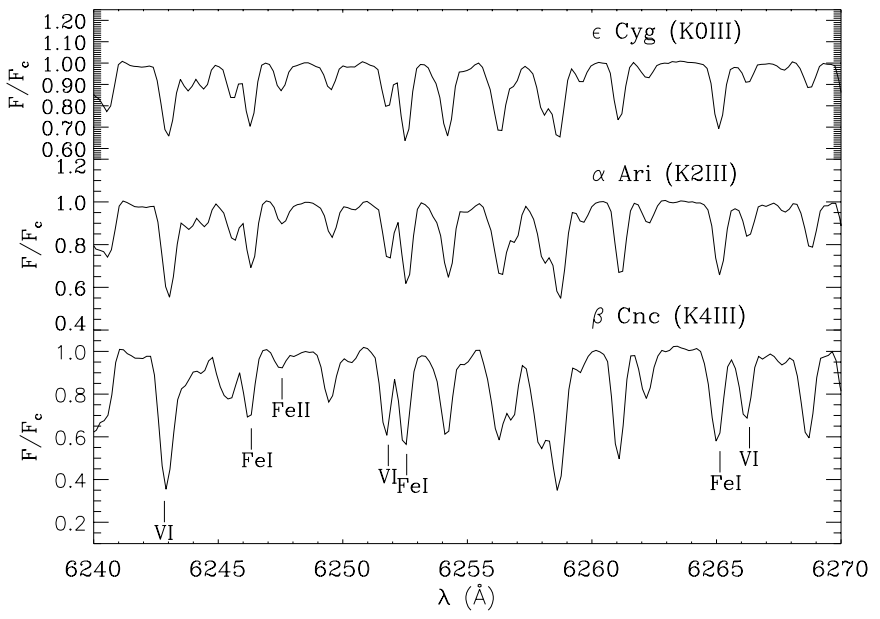

Fig. 1. Sample of standard star spectra in the region around $6250 \AA$.

Gray \& Brown 2001; Hatzes et al. 1998) and $6400 \AA$ (Strassmeier \& Fekel 1990; Strassmeier \& Schordan 2000). We preferred to use lines in the 6100-6200 $\AA$ range because we were able to select a larger number of unblended pairs with separation smaller than $5 \AA$ thus avoiding problems of different setting of the continuum, and less contamination from telluric lines that at our resolution is difficult to remove properly.

Figure 1 displays a portion of the $6200 \AA$ region for a series of spectra of giant stars representative of spectral type from K0III to K4III. From this figure the strengthening of $\mathrm{Fe}_{\mathrm{I}}$ and $\mathrm{V}_{\mathrm{I}}$ lines with decreasing temperature is evident, while the $\lambda 6247$ Fe II shows the opposite behaviour. Furthermore, the growth of low-excitation lines (like those of $\mathrm{V}_{\mathrm{I}}$ ) is faster than that of iron lines. Altogether we identify 15 spectral lines forming 10 pairs suitable for line-depth ratios. These lines were identified through the solar spectral atlas (Moore et al. 1966), choosing the unblended lines. The only exception is the $\lambda 6243 \mathrm{~V}_{\mathrm{I}}$ line that is indeed composed of two very close V I lines of comparable intensities and with the same temperature dependence that appear as a single line at our resolution. Line identification and excitation potential, $\chi$, taken from Moore et al. (1966) and Bashkin \& Stoner (1975) are listed in Table 3.

\subsection{Measurement of line depth}

The lines for each ratio are chosen to be close together in order to minimize errors in choosing the continuum. The lowest five points in the core of each measured line were fitted with a cubic spline and the minimum of this cubic polynomial was taken as the line depth. Writing the line depth $d$ as

$d=\frac{S_{\mathrm{c}}-S_{\mathrm{b}}}{S_{\mathrm{c}}}=1-\frac{S_{\mathrm{b}}}{S_{\mathrm{c}}}$

where $S_{\mathrm{c}}$ and $S_{\mathrm{b}}$ are the signals in ADU (Analog to Digital Units) or in photons of continuum and bottom of the line, respectively, the fractional error on $d$ can be expressed as

$\frac{\sigma_{d}}{d}=\frac{\sigma \frac{S_{\mathrm{b}}}{S_{\mathrm{c}}}}{d}=\frac{1-d}{d} \sqrt{\frac{1}{S_{\mathrm{b}}}+\frac{1}{S_{\mathrm{c}}}}$.
Table 3. Spectral lines used for LDR.

\begin{tabular}{crc}
\hline \hline $\begin{array}{c}\lambda \\
(\AA)\end{array}$ & Element & $\begin{array}{c}\chi \\
(\mathrm{eV})\end{array}$ \\
\hline 6199.19 & $\mathrm{~V}_{\mathrm{I}}$ & 0.29 \\
6200.32 & FeI & 2.61 \\
6210.67 & $\mathrm{ScI}$ & 0.00 \\
6215.15 & FeI & 4.19 \\
6215.22 & TiI & 2.69 \\
6216.36 & $\mathrm{~V}_{\mathrm{I}}$ & 0.28 \\
6243.11 & $\mathrm{~V}_{\mathrm{I}}$ & 0.30 \\
6246.33 & FeI & 3.60 \\
6247.56 & FeII & 3.89 \\
6251.83 & $\mathrm{~V}_{\mathrm{I}}$ & 0.29 \\
6252.57 & FeI & 2.40 \\
6265.14 & FeI & 2.18 \\
6266.33 & $\mathrm{~V}_{\mathrm{I}}$ & 0.28 \\
6268.87 & $\mathrm{~V}_{\mathrm{I}}$ & 0.30 \\
6270.23 & FeI & 2.86 \\
6274.66 & $\mathrm{~V}_{\mathrm{I}}$ & 0.27 \\
\hline
\end{tabular}

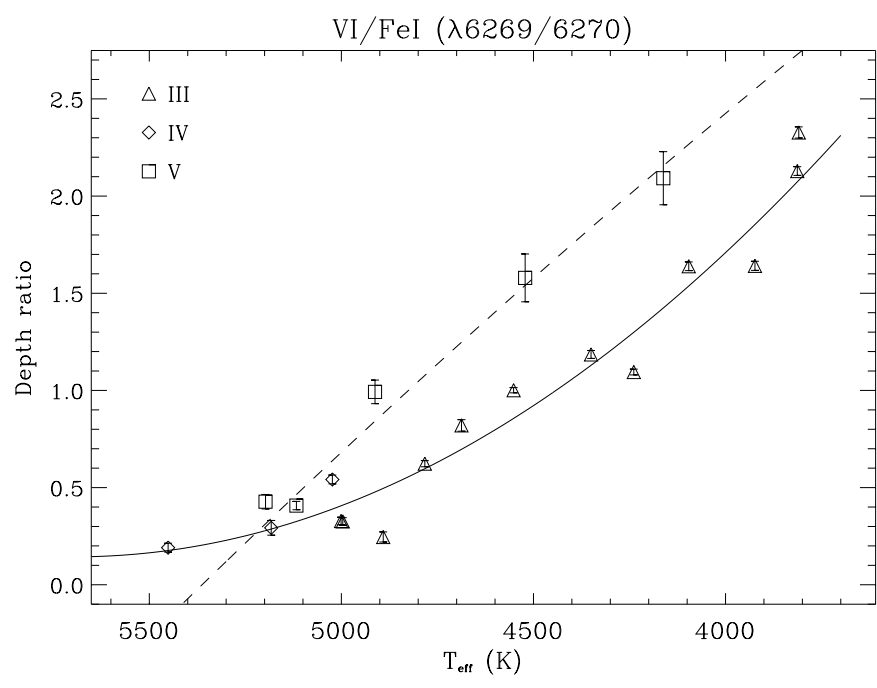

Fig. 2. An example of LDR as a function of effective temperature. Different symbols refer to different luminosity classes. The solid line is the polynomial fit to evolved star LDR; the dashed line represents the fit to main sequence LDR.

This relation has been used to evaluate observational errors on line-depth ratios.

\section{The temperature line-depth ratio calibration}

Using the measured depths we are now able to construct temperature line-depth ratio (LDR) calibration for the different line pairs. We select the $\lambda 6269 \mathrm{~V} \mathrm{I}-\lambda 6270 \mathrm{Fe}_{\mathrm{I}}$ to illustrate the analysis. The other nine pairs are treated in a similar way. The plot of the observed LDR (Fig. 2) versus effective temperature displays two separate dependences: the main-sequence star dependence (squares) and the evolved star dependence, sub-giants (circles) and giants (triangles). For this line pair a significant gravity effect on the LDR is apparent.

In principle we need to determine the temperature variation of active giants as well as main-sequence active stars, so we 


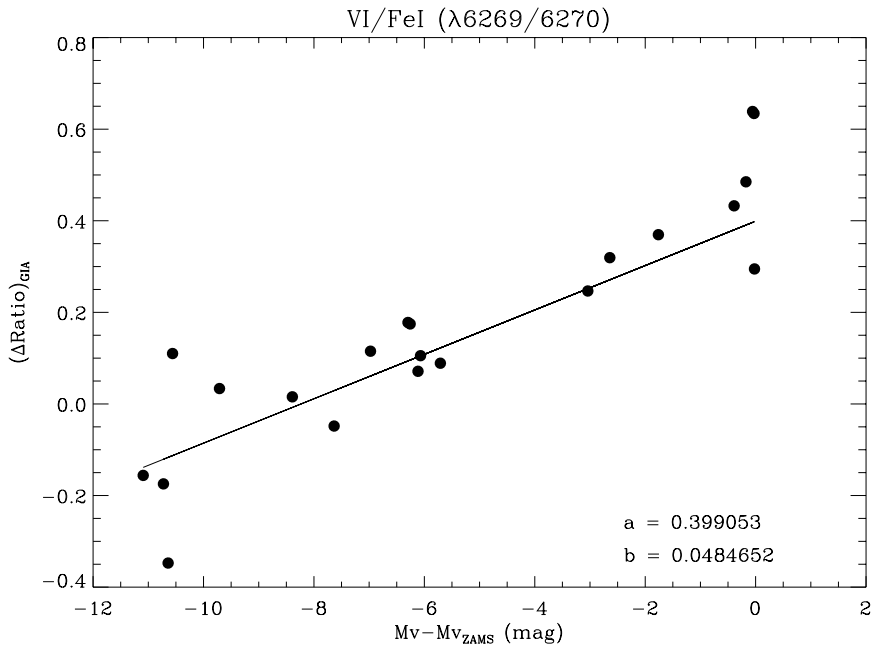

Fig. 3. Residuals of LDRs with respect to the polynomial fit to MS data of Fig. 2 plotted as a function of the gravity indicator $\Delta M_{\mathrm{V}}$ (dots). The continuous line represents a linear fit to the data.

need to set appropriate temperature scales. To use all the observations we have in hand, we proceeded in the following way: (i) first we defined separated temperature scales by doing loworder polynomial fits through main sequence and giant stars, (ii) then we determined the difference of the observed LDR with respect to the fits. Defining as gravity index the absolute magnitude difference $\Delta M_{\mathrm{V}}$ with respect to the ZAMS magnitude for the star's temperature we plot in Fig. 3 the LDR difference with respect to the giant fit, $\Delta$ Ratio $_{\mathrm{GIA}}$, and in Fig. 4 the LDR difference for the MS fit, $\Delta$ Ratio $_{M S}$, as a function of $\Delta M_{\mathrm{V}}$. In both cases a clear correlation is apparent. We have fitted this dependence with a linear regression function (continuous line in Figs. 3 and 4). The absolute-magnitude corrected line-depth ratio for main sequence and giant are then

$\mathrm{LDR}_{\mathrm{MS}, \mathrm{G}}=\mathrm{LDR}-\left(a_{\mathrm{MS}, \mathrm{G}}+b_{\mathrm{MS}, \mathrm{G}} \Delta M_{\mathrm{V}}\right)$

with $b$ and $a$ the slope and the intercept of regression line of $\Delta$ Ratio on $\Delta M_{\mathrm{V}}-$ magnitude correlation lines. With few exceptions, the scatter of the points around the correlation line is consistent with the error of line-depth ratio determination, thus giving confidence in the correction procedure and in the adoption of $\Delta M_{\mathrm{V}}$ as the gravity index.

Plots of the temperature calibration as a function of corrected LDRs are displayed in Figs. 5 and 6. Data points for stars of different luminosity class mix very well, leading to welldefined unique correlations, alternatively applicable to MS and Giant stars. The spread around the polynomial fits (continuous lines in the figures) is greatly reduced and is consistent with the uncertainties and errors in the temperature and LDR determination. The rms of the fits in this case are of $89 \mathrm{~K}$ and $73 \mathrm{~K}$ for MS and Giant-stars calibrations, respectively. This values are comparable with the uncertainty on the setting of the temperature scale (see e.g. Gray 1992). Similar values are found for most of the LDRs.

New low-order polynomial fits were placed through the plots of temperature versus LDRs to obtain separate temperature calibrations for MS and giant stars. Final fits of

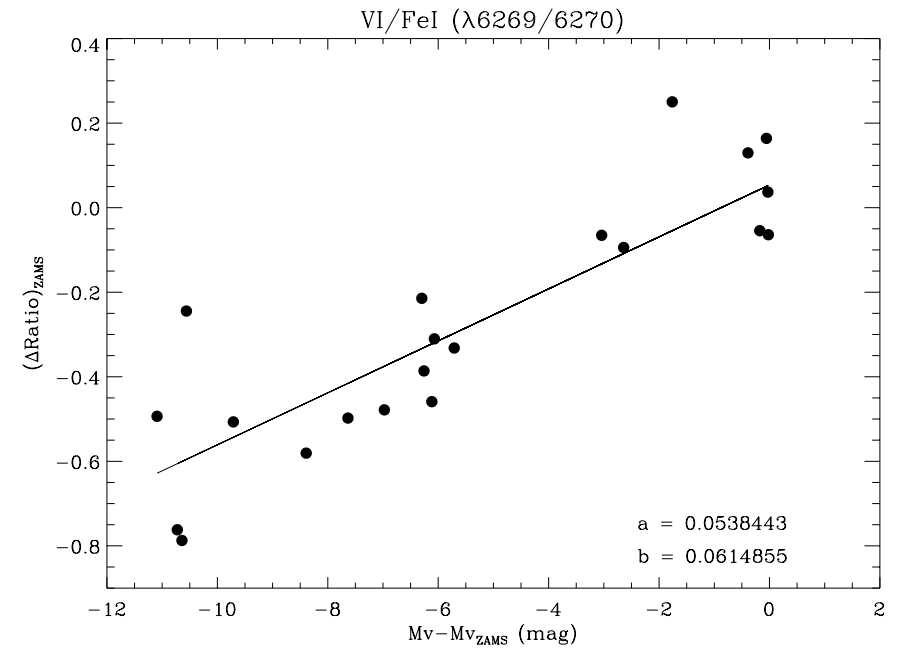

Fig. 4. Residuals of LDRs with respect to the polynomial fit to Giantstars data of Fig. 2 plotted as a function of the gravity indicator $\Delta M_{\mathrm{V}}$ (dots). The continuous line represents a linear fit to the data.

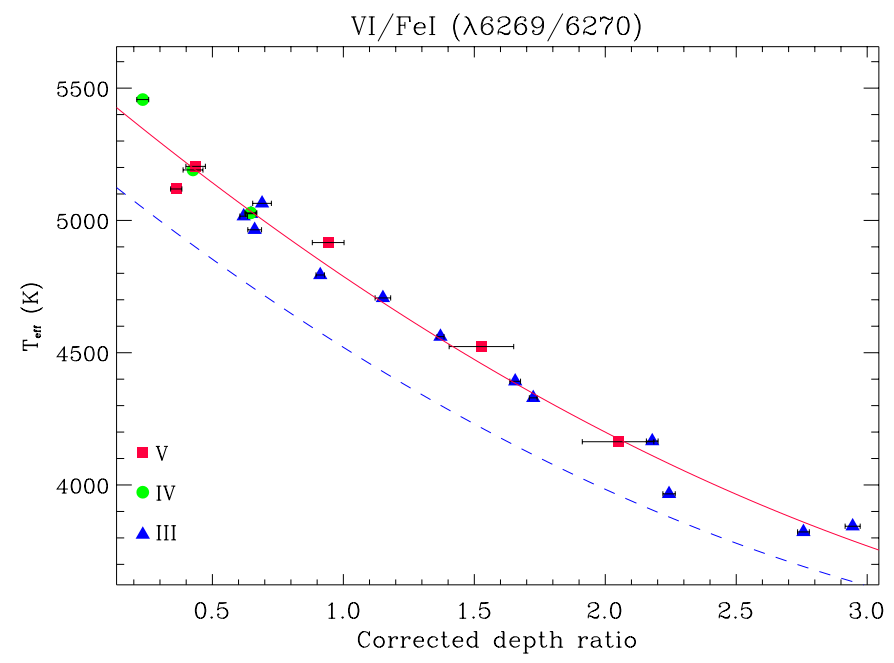

Fig. 5. Effective temperature as a function of corrected LDR for MS stars. The solid line represents a polynomial fit to all data. The dotted line is the polynomial fit to LDR data corrected for Giant-star calibration (solid line in Fig. 6).

temperature versus LDR for the other line pairs are illustrated in Fig. 7. Some of the line pair ratios display a clear gravity effect like the $\lambda 6211 \mathrm{ScI}-\lambda 6215 \mathrm{Fe}+\mathrm{Ti}$ or the $\lambda 6275 \mathrm{VI}-\lambda 6270 \mathrm{Fe} \mathrm{I}$, that becomes very strong for the two ratios $\lambda 6243 \mathrm{~V} \mathrm{I}-\lambda 6247 \mathrm{Fe}$ II and $\lambda 6246 \mathrm{Fe}_{\mathrm{I}}-\lambda 6247 \mathrm{Fe}$ II. Other ratios, like the $\lambda 6252 \mathrm{VI}-\lambda 6253 \mathrm{Fe}_{\mathrm{I}}$ or $\lambda 6266 \mathrm{~V} \mathrm{I}-\lambda 6265 \mathrm{Fe} \mathrm{I}$, do show a very marginal gravity effect. In all cases we have made separate temperature calibrations.

For the two LDRs involving the $\lambda 6247 \mathrm{Fe}$ II line, the gravity dependence is so high that the correction method described above could not be applied to derive average relations for the MS stars and for Giant stars, using all standard stars.

In order to measure the temperature sensitivity of each linedepth ratio we have calculated the slopes of the polynomial fits $\frac{\mathrm{d} T}{\mathrm{~d} r}$ at temperatures of $4500 \mathrm{~K}, 5000 \mathrm{~K}$ and $5500 \mathrm{~K}$ (typical of most of active RS CVn binaries), for a 0.01 variation in LDR, 


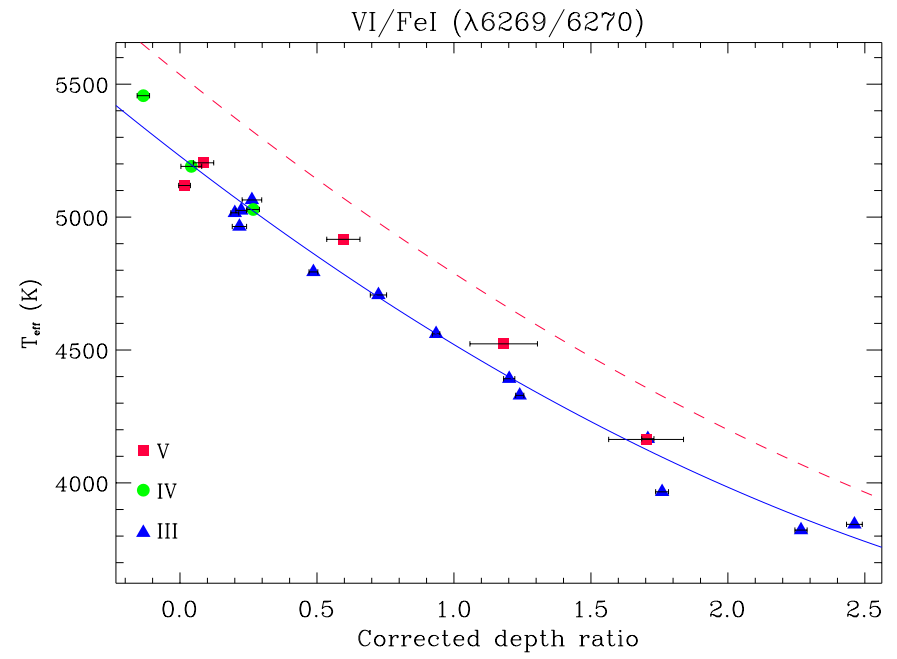

Fig. 6. Effective temperature as a function of corrected LDR for Giant stars. The solid line represents a polynomial fit to all data. The dotted line is the polynomial fit to LDR data corrected for MS-star calibration (solid line in Fig. 5).

Table 4. Temperature sensitivity of LDRs.

\begin{tabular}{rrrr|rrr}
\hline \hline Lines pair & \multicolumn{3}{c}{$\Delta T$ for $\Delta r=0.01$} & \multicolumn{3}{c}{$\Delta T$ for $\Delta r=0.01$} \\
& $4500 \mathrm{~K}$ & $5000 \mathrm{~K}$ & $5500 \mathrm{~K}$ & $4500 \mathrm{~K}$ & $5000 \mathrm{~K}$ & $5500 \mathrm{~K}$ \\
\hline & \multicolumn{3}{c}{ DWARFS } & & \\
$6199 / 6200$ & 10.4 & 11.4 & 14.1 & 11.7 & 13.0 & 15.3 \\
$6211 / 6215$ & 10.5 & 12.4 & - & 10.4 & 12.3 & - \\
$6216 / 6215$ & 18.4 & 15.3 & 20.2 & 18.8 & 15.6 & 20.5 \\
$6243 / 6246$ & 9.1 & 11.1 & 14.3 & 9.9 & 12.1 & 15.7 \\
$6243 / 6247$ & - & 1.2 & 91.8 & 2.0 & 5.4 & 6.0 \\
$6246 / 6247$ & - & 1.3 & 87.7 & 7.2 & 20.1 & 11.8 \\
$6252 / 6253$ & 22.1 & 23.5 & 25.0 & 21.9 & 23.2 & 24.7 \\
$6266 / 6265$ & 17.1 & 18.1 & 19.1 & 16.2 & 16.5 & 16.8 \\
$6269 / 6270$ & 5.8 & 7.6 & 11.0 & 6.0 & 8.2 & 12.8 \\
$6275 / 6270$ & 8.5 & 9.7 & 11.4 & 8.6 & 9.9 & 11.8 \\
\hline
\end{tabular}

which represents the typical uncertainty for the LDR determination in well exposed spectra. From single LDR-dependence we derived typical sensitivities of 10-20 K, and in some case even smaller, for a 0.01 variation in $r$ (see Table 4).

\section{Line depth ratio and temperature variation of the active stars}

Line-depth ratios variations are analysed separately for each active star considered. Since we have 10 different pairs of lines we have first analysed each ratio as a function of the rotational phase for the three stars and then we have transformed the LDRs in temperature. Some line pairs could not be used for our active star due to the non-negligible $v \sin i$ of the targets that causes blending of the lines that are too close in wavelength. Temperature values from all pairs for each spectrum were finally combined to yield an average temperature value, thus reducing the temperature errors. We have excluded from
Table 5. Average surface temperature of VY Ari.

\begin{tabular}{ccl}
\hline \hline $\begin{array}{c}\text { HJD } \\
(+2451000)\end{array}$ & $\phi$ & $\begin{array}{c}<T_{\text {eff }}> \\
(\mathrm{K})\end{array}$ \\
\hline 856.4761 & 0.029 & $4799 \pm 16$ \\
857.4544 & 0.090 & $4767 \pm 9$ \\
859.4971 & 0.216 & $4760 \pm 13$ \\
860.4516 & 0.275 & $4798 \pm 5$ \\
861.4502 & 0.336 & $4822 \pm 6$ \\
862.4862 & 0.400 & $4821 \pm 13$ \\
863.5280 & 0.465 & $4850 \pm 4$ \\
864.5596 & 0.528 & $4895 \pm 4$ \\
865.4910 & 0.586 & $4916 \pm 21$ \\
866.5074 & 0.649 & $4899 \pm 7$ \\
867.5400 & 0.712 & $4850 \pm 12$ \\
913.3357 & 0.539 & $4855 \pm 8$ \\
915.3436 & 0.663 & $4909 \pm 2$ \\
916.3325 & 0.724 & $4888 \pm 9$ \\
917.4153 & 0.791 & $4881 \pm 4$ \\
\hline
\end{tabular}

the means those LDR values that are outside the $3 \sigma$ box around the mean value.

Temperature variation curves have been obtained for each active star by folding in phase individual $<T_{\text {eff }}>$ data, analogously to what is performed with photometric measurements.

As can be seen from Tables 5-7, our $<T_{\text {eff }}>$ measurements span a time range of 4-5 stellar rotations, but it has been shown that the big spots observed in very active stars have typical lifetimes of several rotations. For a set of four spotted RS CVn stars, Henry et al. (1995) observed individual spot lifetimes between 0.5 years and over 6 years. Spot lifetimes in the same range were also found for other RS CVn stars, including VY Ari, IM Peg and HK Lac (Strassmeier \& Bopp 1992; Strassmeier et al. 1994; Oláh et al. 1997; Strassmeier et al. 1997; Frasca et al. 1998).

\subsection{VY Ari}

Due to blends induced by the rotational broadening and by the crowding related to the spectral type of the visible component of VY Ari (K3-4 V-IV) only seven combinations of LDRs could be used for the temperature variation study. The useful measured line-depth ratios of VY Ari are plotted in Fig. 8 as a function of the rotational phase, computed from the following ephemeris

$\mathrm{HJD}_{\phi=0}=2451856.0+16$ d.1996 $\times E$,

where the rotational period is taken from Strassmeier et al. (1997) and the epoch of zero phase corresponds to the first observing date (November 7th, 2000) at noon.

All LDRs show a clear modulation with the rotational phase with a maximum around $0^{\mathrm{p}} .2$ and a minimum around $0^{\mathrm{p}} .6$. The latter value, for all the LDRs, corresponds to the maximum temperature value as displayed on the right side scale. The amplitude variation of the LDRs ranges from $16 \%$ for the $\lambda 6252 \mathrm{~V} \mathrm{I}-\lambda 6253 \mathrm{Fe}$ I ratio to $46 \%$ for the $\lambda 6266 \mathrm{~V} \mathrm{I}-\lambda 6265 \mathrm{Fe} \mathrm{I}$ ratio which appears to be the more sensitive to the temperature. 

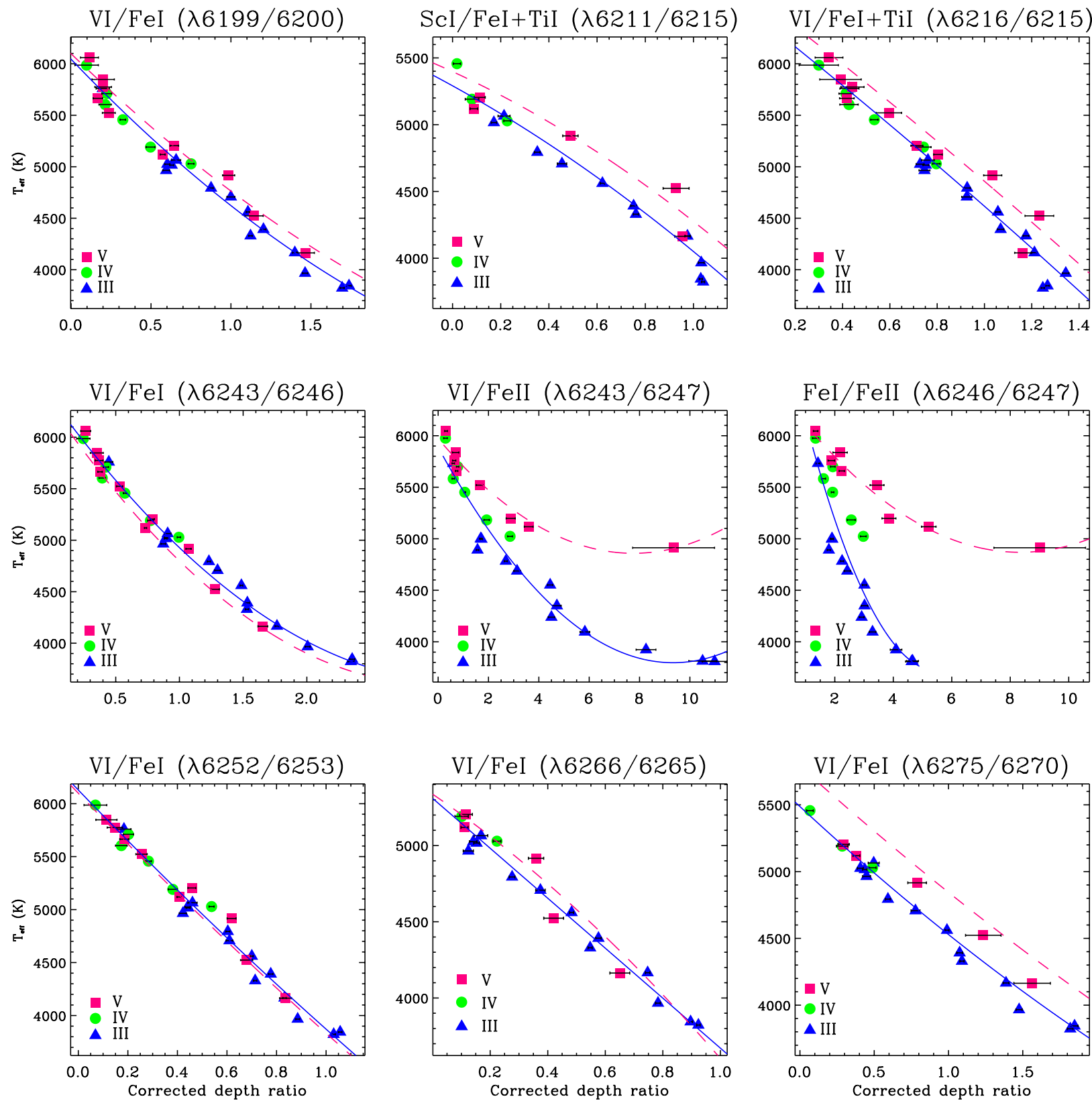

Fig. 7. $T_{\text {eff }}-$ LDR calibrations for Giant stars. The meaning of symbols and curves is as in Figs. 5 and 6.

These variations are well above (3-5 times) the average errors, which are determined according to error propagation rule as:

$\frac{\sigma_{r}}{r}=\sqrt{\left(\frac{\sigma_{d_{1}}}{d_{1}}\right)^{2}+\left(\frac{\sigma_{d_{2}}}{d_{2}}\right)^{2}}$,

where $d_{1}$ and $d_{2}$ are the line depths and $\sigma_{d_{1}}$ and $\sigma_{d_{2}}$ are the corresponding absolute errors as fixed by the $S / N$ ratio for the measured continuum and central line flux.

Temperature values derived from the LDR- $T_{\text {eff }}$ calibration from each pair are plotted in Fig. 9 using different symbols.

The temperature variation derived from all the LDRs displays a common behaviour, with a spread consistent with error estimate. Apart from the very similar shape, the temperature curves derived from different LDRs display a small offset one with respect to the other. Since we are mainly interested in the temperature variation, not in its absolute value, we have evaluated the average $T_{\text {eff }}$ from all curves and have shifted each $T_{\text {eff }}$ curve of the offset needed to make its average level equal to the average from all curves. These temperature offsets are in the range $20-50 \mathrm{~K}$ and may be due to some residual gravity dependence that has not been completely accounted for by the correction procedure or to the influence of some other physical parameter that has a minor effect on the LDR. This scaling procedure, applied to several LDRs, can statistically compensate for such effects and, also in the present case with only 6 or 7 useful LDRs, will give also a good evaluation of the absolute temperature scale that, however, has its intrinsic setting uncertainty of a few tens of Kelvin degrees (see e.g. Gray 1992).

We have then derived an average temperature variation by making a weighted mean of the values obtained from each 


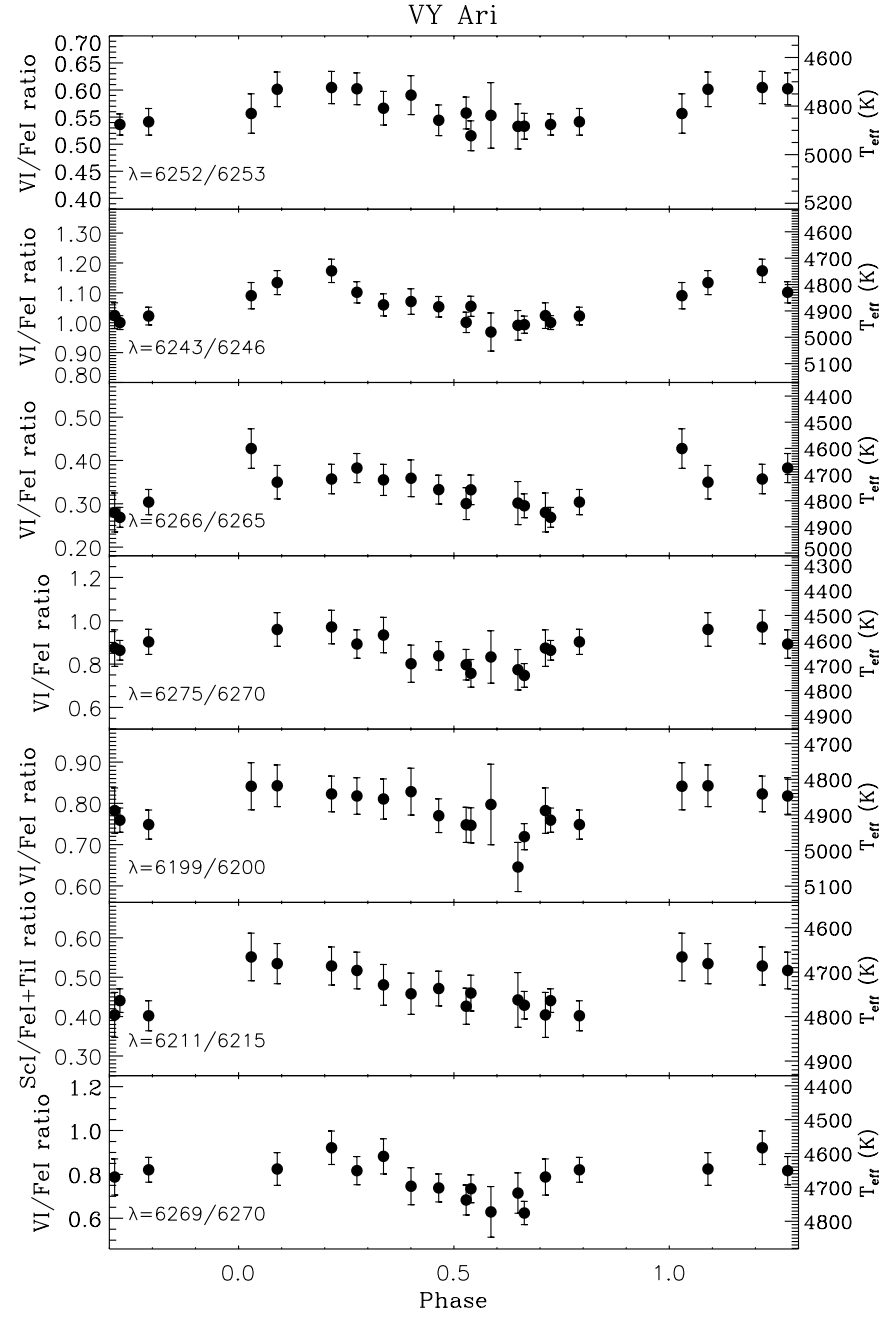

Fig. 8. LDRs of VY Ari versus rotational phase as computed according to Eq. (5). The temperature scale for each ratio is displayed on the right side of the boxes.

spectrum. The weighted mean has been given by:

$<T_{\mathrm{eff}}>=\frac{\sum_{i=1}^{n} w_{i} T_{i}}{\sum_{i=1}^{n} w_{i}}$,

and the corresponding error has been computed as:

$\sigma_{<T_{\text {eff }}>}=\sqrt{\frac{\sum_{i=1}^{n} w_{i}\left(T_{i}-<T_{\text {eff }}>\right)^{2}}{(n-1) \sum_{i=1}^{n} w_{i}}}$,

where $T_{i}=T\left(r_{i}\right)$ is the effective temperature obtained from the $i$ th line-depth ratio, and $w_{i}=1 / \sigma_{T_{i}}^{2}$ is the corresponding statistical weight.

The final temperature variation ranges from $4739 \mathrm{~K}$ to $4916 \mathrm{~K}$, i.e. with a $\Delta<T_{\text {eff }}>=177 \mathrm{~K}$. As can be seen in Table 5, $\sigma_{<T_{\text {eff }}>}$ errors are typically of a few Kelvin degree.

\subsection{IM Peg}

For IM Peg we were also able to use seven LDRs, but with some differences, as displayed in Fig. 10, where single LDR values are plotted as function of the rotational phase.
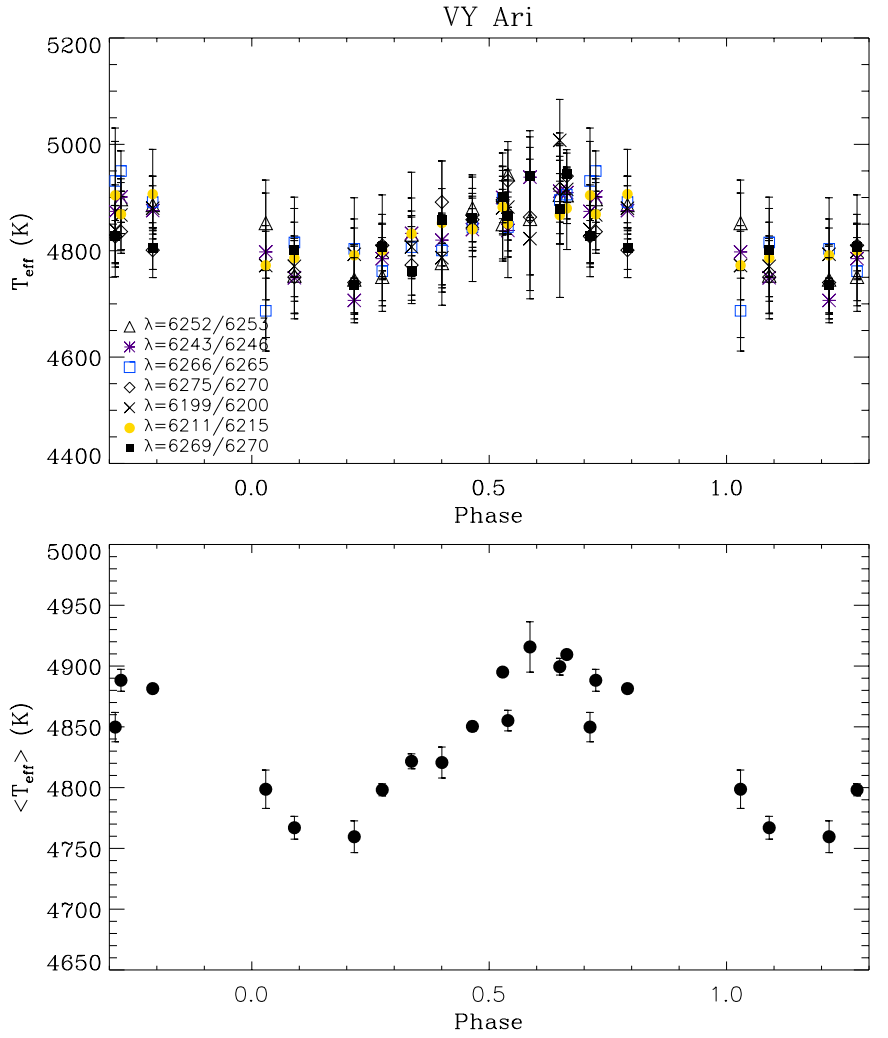

Fig. 9. Temperature curves of VY Ari obtained from the LDRs in Fig. 8 (upper panel). Different symbols have been used for the different ratios. The average effective temperature $<T_{\text {eff }}>$ as a function of rotational phase is displayed in the lower panel.

Phases are reckoned from the ephemeris given by Strassmeier et al. (1997)

$\mathrm{HJD}_{\phi=0}=2443734.0+24.494 \times E$.

In this case the amplitude of the LDR variation is smaller and the average behaviour is more noisy and not well defined.

IM Peg actually represents a proper test case for the application and reliability of the method. Its rotational broadening $\left(26.5 \mathrm{~km} \mathrm{~s}^{-1}\right)$ is a bit larger than our spectral resolution so that the Doppler shifts of the bumps produced by the spots could be partially resolved in our spectra, and for sure are responsible for the larger noise. As a matter of fact, Berdyugina et al. (2000) from high resolution spectra $(R=30000-80000)$ were able to obtain surface images with the Doppler-imaging technique.

Notwithstanding this limitation a maximum LDR variation of $37 \%$ is obtained for the $\lambda 6275 \mathrm{~V} \mathrm{I}-\lambda 6270 \mathrm{Fe}$ r ratio.

However, all the LDRs converted to temperature and combined in a single temperature curve, as displayed in Fig. 11, lead to a fairly well-defined temperature variation as a function of the rotational phase. The average curve obtained from the weighted mean (lower panel in Fig. 11) appears still well defined. The temperature maximum, with a value of $4666 \mathrm{~K}$, occurs around phase 0.5 . The full amplitude variation is $\Delta<T_{\text {eff }}>=119 \mathrm{~K}$, corresponding to a $3 \%$ of the determined average temperature value. 


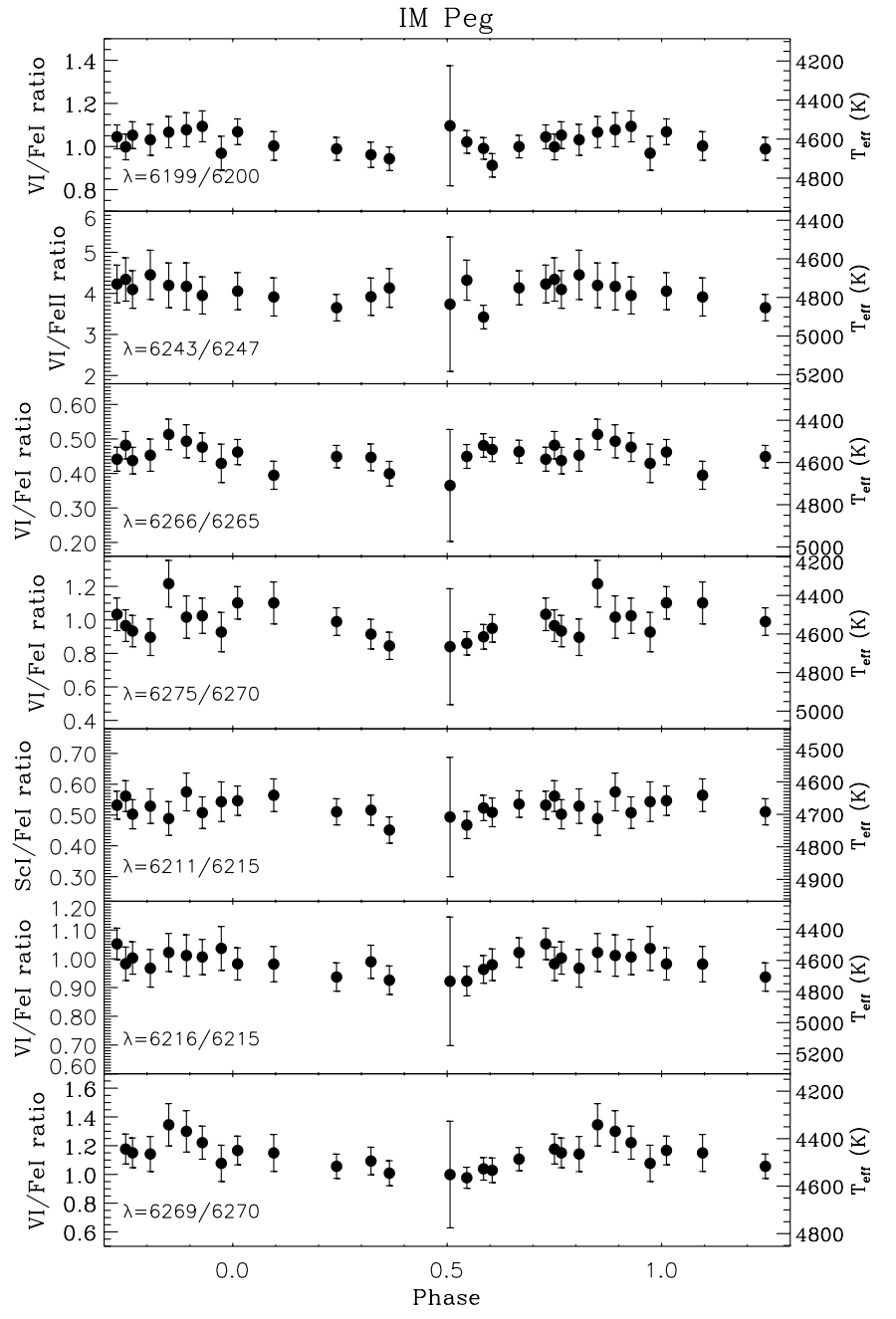

Fig. 10. LDRs of IM Peg versus rotational phase as computed according to Eq. (9). The temperature scale for each ratio is displayed on the right side of the boxes.

\subsection{HK Lac}

For HK Lac we were able to use six LDRs, as displayed in Fig. 12 where single values are plotted as a function of the rotational phase computed from the ephemeris

$\mathrm{HJD}_{\phi=0}=2440017.17+24.4284 \times E$,

which is the orbital ephemeris already given by Gorza \& Heard (1971) with the initial epoch given by Strassmeier et al. (1993) corresponding to inferior conjunction (more massive star closer to the observer).

All the ratios exhibit a well-defined parallel behaviour; even single values have slightly larger errors due to the average lower $S / N$ ratio of the observations.

The largest amplitude variation is displayed by the $\mathrm{V}$ I-Fe II at $6243 \AA$ and $6247 \AA$, with a full variation of $40 \%$. The corresponding temperature range is very similar for all the LDRs, as displayed in Fig. 13 (upper panel) where the temperature values deduced according to the above calibrations are plotted. The spread of the points is consistent with the estimated errors, and the mean curve resulting from weighted average has a well defined variation. The temperature maximum of $4765 \mathrm{~K}$ seems
Table 6. Average surface temperature of IM Peg.

\begin{tabular}{ccl}
\hline \hline $\begin{array}{c}\text { HJD } \\
(+2451000)\end{array}$ & $\phi$ & $\begin{array}{c}<T_{\text {eff }}> \\
(\mathrm{K})\end{array}$ \\
\hline 798.4555 & 0.242 & $4615 \pm 8$ \\
800.4203 & 0.322 & $4608 \pm 4$ \\
801.4724 & 0.365 & $4666 \pm 7$ \\
829.4298 & 0.507 & $4654 \pm 18$ \\
830.3874 & 0.546 & $4645 \pm 19$ \\
831.3436 & 0.585 & $4622 \pm 13$ \\
833.3725 & 0.668 & $4589 \pm 6$ \\
835.3963 & 0.750 & $4569 \pm 9$ \\
856.3316 & 0.605 & $4621 \pm 11$ \\
859.3944 & 0.730 & $4585 \pm 10$ \\
860.2836 & 0.766 & $4606 \pm 9$ \\
861.2989 & 0.808 & $4593 \pm 5$ \\
862.3525 & 0.851 & $4578 \pm 25$ \\
863.3614 & 0.892 & $4547 \pm 6$ \\
865.3533 & 0.973 & $4613 \pm 14$ \\
913.2565 & 0.929 & $4582 \pm 14$ \\
915.2733 & 0.011 & $4574 \pm 7$ \\
917.3364 & 0.096 & $4585 \pm 12$ \\
\hline
\end{tabular}

Table 7. Average surface temperature of HK Lac.

\begin{tabular}{ccl}
\hline \hline $\begin{array}{c}\text { HJD } \\
(+2451000)\end{array}$ & $\phi$ & $\begin{array}{c}<T_{\text {eff }}> \\
(\mathrm{K})\end{array}$ \\
\hline 798.4325 & 0.277 & $4686 \pm 3$ \\
800.4422 & 0.360 & $4716 \pm 15$ \\
801.4907 & 0.402 & $4698 \pm 52$ \\
830.3564 & 0.584 & $4765 \pm 8$ \\
831.3613 & 0.625 & $4720 \pm 12$ \\
833.3913 & 0.708 & $4734 \pm 12$ \\
835.4153 & 0.791 & $4709 \pm 3$ \\
836.4205 & 0.832 & $4689 \pm 12$ \\
856.3085 & 0.646 & $4741 \pm 33$ \\
858.2968 & 0.728 & $4708 \pm 15$ \\
860.2632 & 0.808 & $4724 \pm 19$ \\
861.2775 & 0.850 & $4702 \pm 17$ \\
862.3297 & 0.893 & $4660 \pm 6$ \\
863.3362 & 0.934 & $4682 \pm 8$ \\
865.3329 & 0.016 & $4640 \pm 14$ \\
913.2360 & 0.977 & $4651 \pm 11$ \\
915.2508 & 0.059 & $4656 \pm 2$ \\
916.2584 & 0.101 & $4641 \pm 7$ \\
917.3159 & 0.144 & $4638 \pm 31$ \\
\hline
\end{tabular}

to occur around phase $0^{\mathrm{p}} \cdot 6$. The temperature variation we get is $127 \mathrm{~K}$, corresponding to $\simeq 3 \%$ of the average value.

\section{Discussion}

Only a few active stars investigated until now have shown clear rotational modulation of line-depth ratios (e.g. $\sigma$ Dra, Gray et al. 1992; $\xi$ Boo A, Toner \& Gray 1988). However all these studies have been devoted to young main-sequence stars with an activity degree detectably lower than RS CVn and BY Dra binaries and, consequently, with a temperature variation of only 

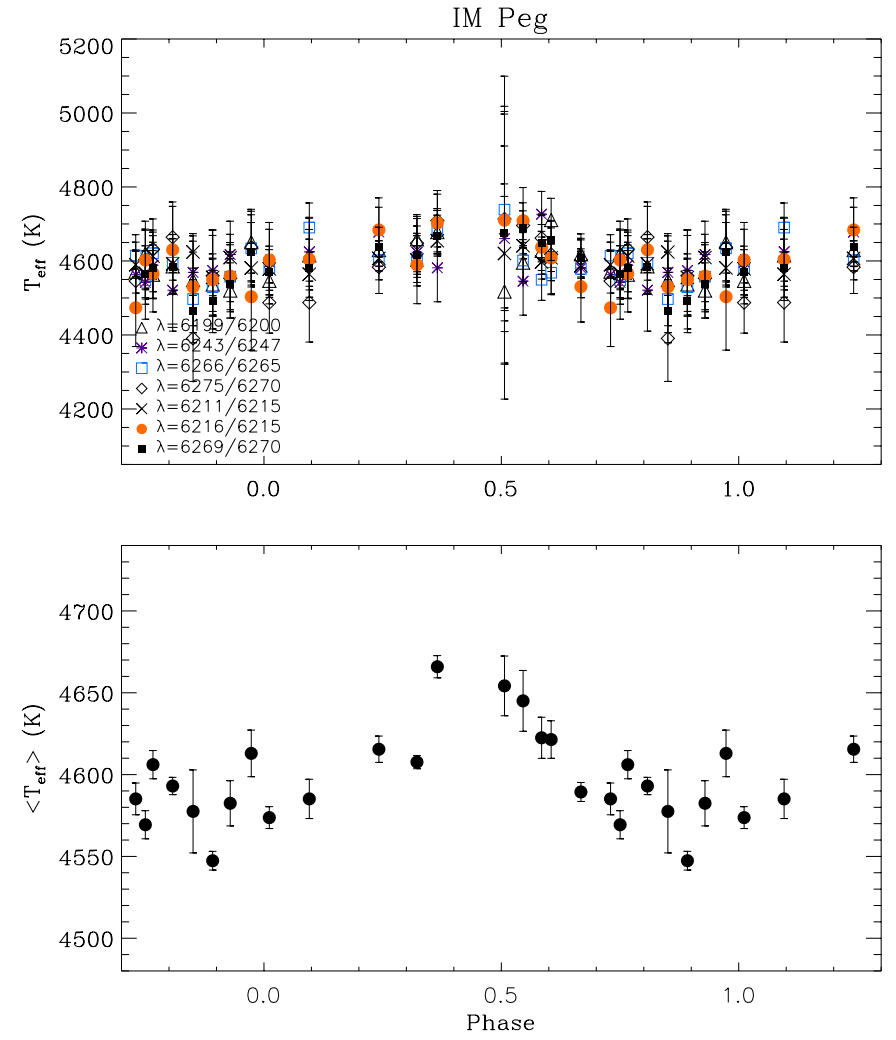

Fig. 11. Temperature curves of IM Peg obtained from the LDRs in Fig. 10 (upper panel). Different symbols have been used for the different ratios. The average effective temperature $<T_{\text {eff }}>$ as a function of rotational phase is displayed in the lower panel.

a few degrees or a bit more. Conversely, many more cases of long-term variation of average temperature have been found and have been attributed to stellar activity cycles similar to the 11-year solar one (e.g. Gray et al. 1996a, 1996b). These detections have been possible thanks to the large number of spectra collected in each season and averaged together with a great improvement of the $S / N$ ratio.

Since the temperature curves we obtained are $T_{\text {eff }}$ averaged over the visible hemisphere, it is not possible to deduce directly the value of the spot temperature because the effect of the filling factor influences also this diagnostic. The dependence of average temperature on the spot relative area is different that of light curves. We can express the hemisphere-averaged temperature as

$T_{\mathrm{m}}=\frac{\int_{\mathrm{disk}} F T \mathrm{~d} A}{\int_{\mathrm{disk}} F \mathrm{~d} A}=\frac{A_{\mathrm{rel}} F_{\mathrm{sp}} T_{\mathrm{sp}}+\left(1-A_{\mathrm{rel}}\right) F_{\mathrm{ph}} T_{\mathrm{ph}}}{A_{\mathrm{rel}} F_{\mathrm{sp}}+\left(1-A_{\mathrm{rel}}\right) F_{\mathrm{ph}}}$,

where $A_{\text {rel }}$ is the projected area of the spot (or spots) relative to the stellar disk, $T_{\mathrm{sp}}$ and $T_{\mathrm{ph}}$ are the temperatures of spot and quiet photosphere, respectively, and $F_{\mathrm{sp}}$ and $F_{\mathrm{ph}}$ are the fluxes emitted per unit area by the spot and the photosphere at the continuum of observation wavelength, respectively.

Equation (11) can be also written as

$T_{\mathrm{m}}=\frac{\gamma T_{\mathrm{sp}}+T_{\mathrm{ph}}}{\gamma+1}$

where $\gamma=\frac{A_{\text {rel }}}{1-A_{\text {rel }}} \frac{F_{\mathrm{sp}}}{F_{\mathrm{ph}}}$.

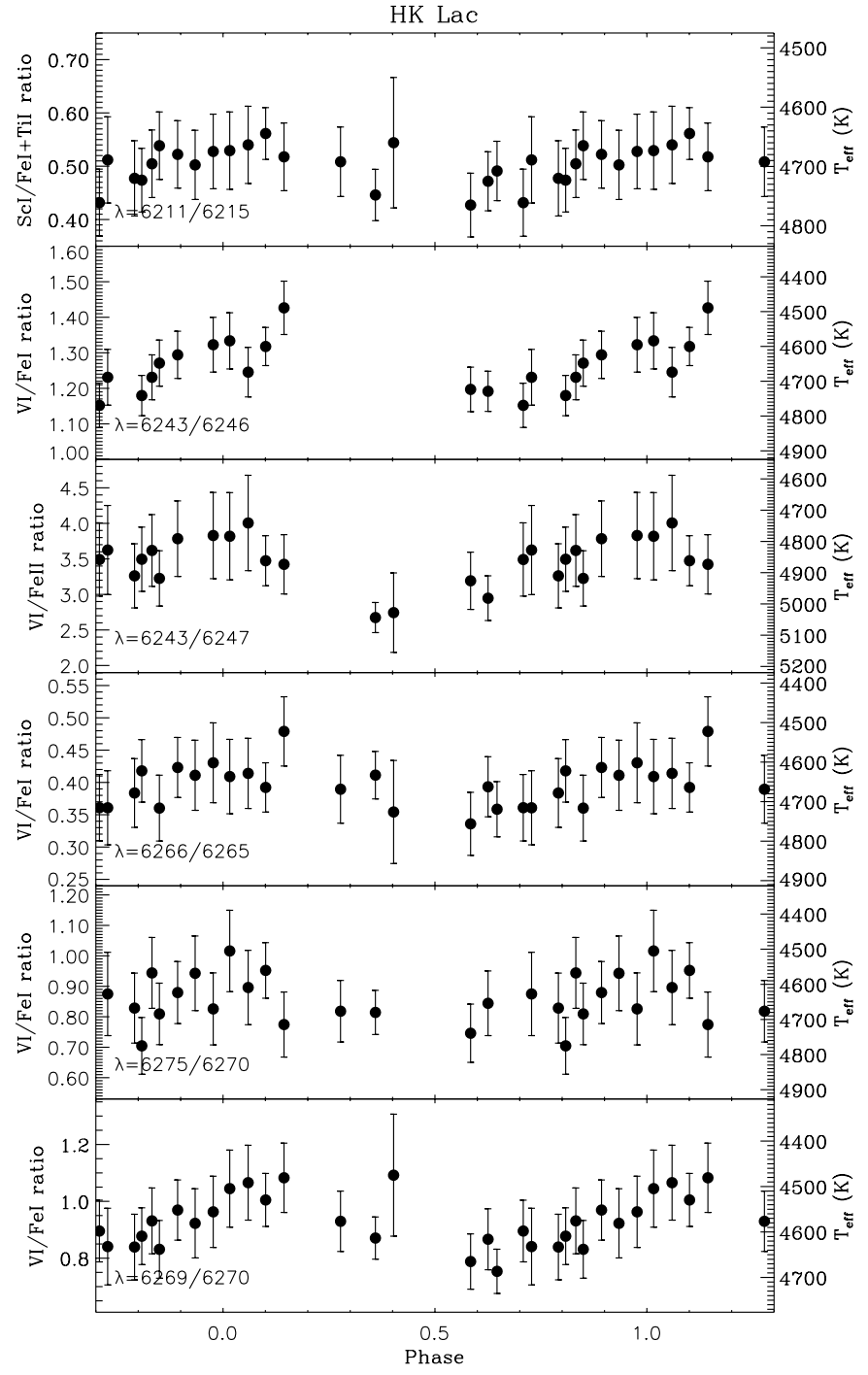

Fig. 12. LDRs of HK Lac versus rotational phase as computed according to Eq. (10). The temperature scale for each ratio is displayed on the right side of the boxes.

If the spot is very cool, its contribution to the mean temperature is negligible because the flux ratio $\frac{F_{\mathrm{sp}}}{F_{\mathrm{ph}}}$ goes very rapidly to zero (much faster than $\frac{T_{\mathrm{sp}}}{T_{\mathrm{ph}}}$ ) with the decrease of $T_{\mathrm{sp}}$. Then $\gamma$ tends to zero and the average temperature tends to equal the photospheric one, so that a very large spot area would be required to account for the observed temperature variation.

In the opposite case, when $\frac{T_{\mathrm{sp}}}{T_{\mathrm{ph}}}$ approaches unity, the average temperature $T_{\mathrm{m}}$ is not appreciably changed by the passage of spots over the visible hemisphere. Again, very large spots are needed to reproduce the observed $T_{\mathrm{m}}$ variation. Then, there is a limited range for physically reliable solutions. In particular, given an observed variation amplitude $\Delta T_{\text {eff }}$, there is a minimum spot area that can reproduce the observations.

As a first approximation we can estimate this minimum spotted area assuming that it is concentrated in only one hemisphere, and its passage causes the observed temperature decrease $\Delta T_{\text {eff. }}$. The maximum temperature value is assumed as the effective unspotted temperature $\left(T_{\mathrm{ph}}\right)$ of the star. 

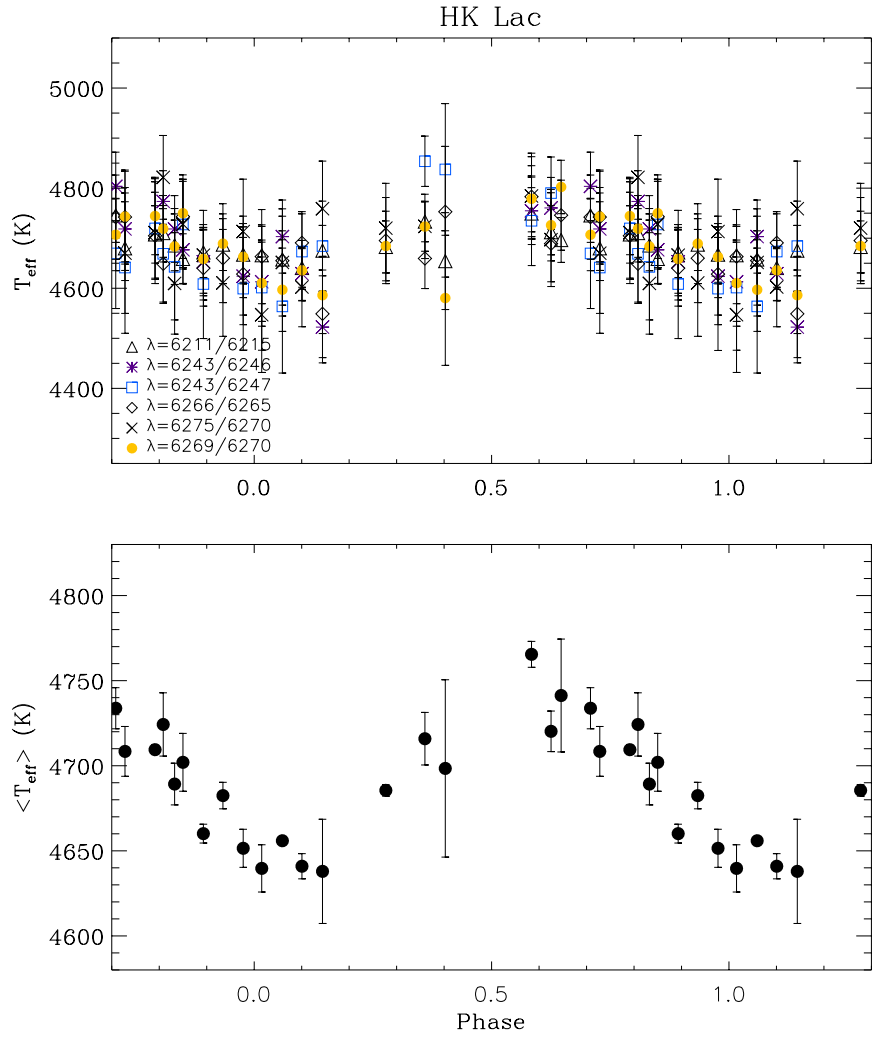

Fig. 13. Temperature curves of HK Lac obtained from the LDRs in Fig. 12 (upper panel). Different symbols have been used for the different ratios. The average effective temperature $<T_{\text {eff }}>$ as a function of rotational phase is displayed in the lower panel.

Starting from relation 12 , we have numerically searched in the $\frac{T_{\mathrm{sp}}}{T_{\mathrm{ph}}}-A_{\text {rel }}$ plane the solution for the minimum $A_{\text {rel }}$ value compatible with the observed $\Delta T_{\text {eff }}$ for each program star. The flux ratio $\frac{F_{\mathrm{sp}}}{F_{\mathrm{ph}}}$ has been evaluated as the ratio of the Planck functions at the average wavelength of observations, $\frac{B\left(\lambda, T_{\mathrm{p} p}\right)}{B\left(\lambda, T_{\mathrm{ph}}\right)}$.

We have no information on the maximum magnitude at the time of observation with respect to the unspotted magnitude of our program stars, however, we would like to remark that the maximum values of temperature we determined for all the three active stars are in very good agreement with the effective temperature reported in the literature. This proves the power of LDRs as temperature indicators as already pointed out by previous works (Gray 1989; Gray \& Johanson 1991). The largest uncertainty in this task, as stressed by Gray (1989), is given by the setting of the absolute scale of temperature for a set of standard stars, while it is differentially possible to put in a growing temperature order each observed star with a precision of about $10 \mathrm{~K}$, which amounts to about one hundredth of spectral subclass or 0.004 mag on $B-V$ color index (Gray 1989; Gray \& Johanson 1991).

Figure 14 displays the solutions in the plane $\frac{T_{\mathrm{sp}}}{T_{\mathrm{ph}}}-A_{\text {rel }}$ for VY Ari with $T_{\mathrm{ph}}=4916 \mathrm{~K}$ and $\Delta T_{\text {eff }}=177 \mathrm{~K}$. The plot shows the parabolic shape of the family of solutions, which has a minimum fractional area $41 \%$ of the projected disk (corresponding to a radius of $40^{\circ}$ for a single circular spot passing at the disk center) for a temperature ratio of 0.82 . This constitutes a lower

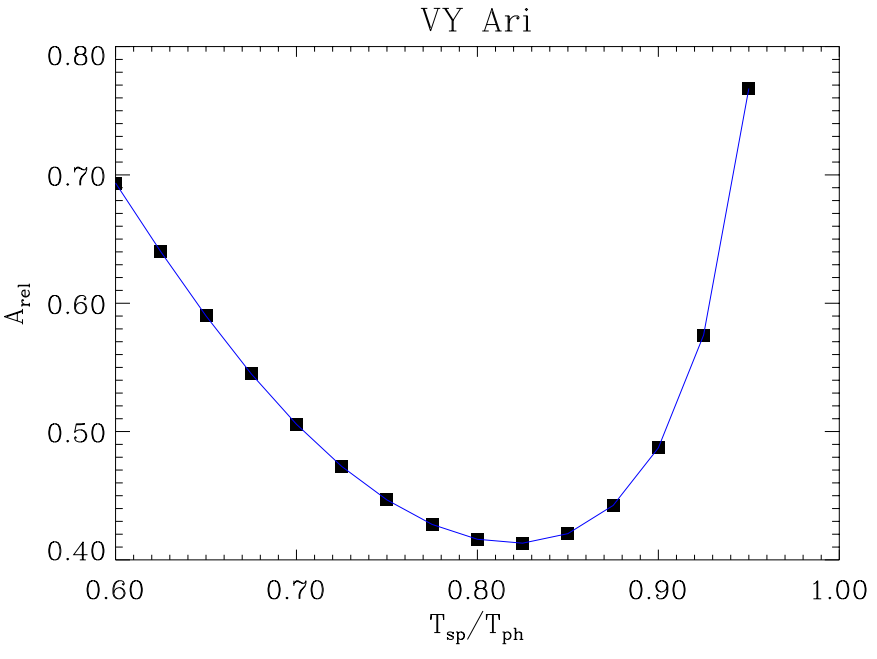

Fig. 14. Solutions of VY Ari $T_{\text {eff }}$ curve for different spots temperature $\frac{T_{\mathrm{sp}}}{T_{\mathrm{ph}}}$. A lower limit for the fractional area $A_{\text {rel }}$ of about $41 \%$ for $\frac{T_{\mathrm{sp}}}{T_{\mathrm{ph}}} \simeq$ 0.82 is apparent.

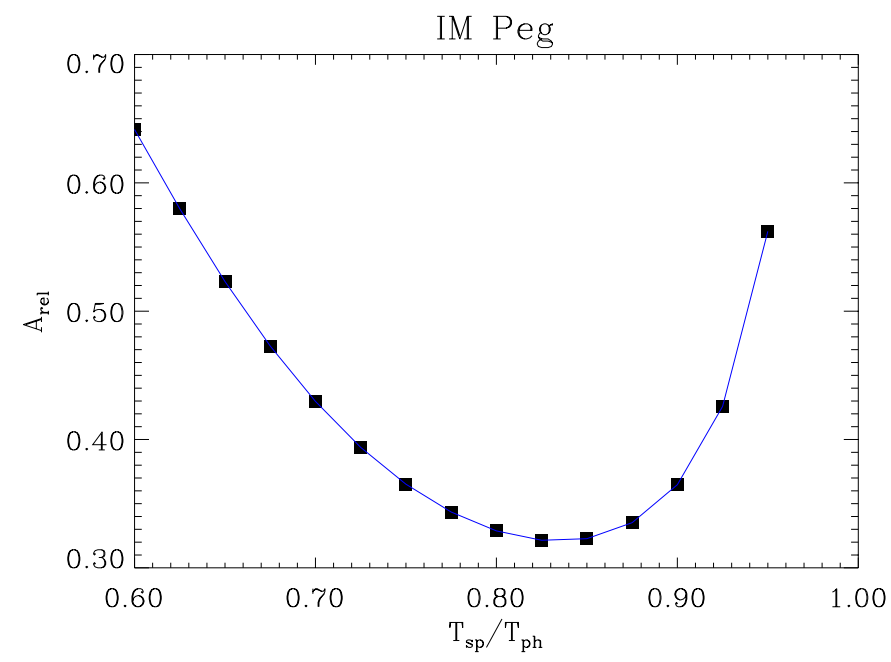

Fig. 15. Solutions of IM Peg $T_{\text {eff }}$ curve for different spots temperature $\frac{T_{\mathrm{sp}}}{T_{\mathrm{ph}}}$. A lower limit for the fractional area $A_{\text {rel }}$ of about $32 \%$ for $\frac{T_{\mathrm{sp}}}{T_{\mathrm{ph}}} \simeq$ 0.84 is apparent.

limit for the spot filling factor, and an average temperature for the spotted area $T_{\mathrm{sp}}=4030 \mathrm{~K}$.

The solutions in the plane $\frac{T_{\mathrm{sp}}}{T_{\mathrm{ph}}}-A_{\text {rel }}$ for IM Peg are shown in Fig. 15. A lower limit for the projected fractional area $A_{\text {rel }}$ of $\simeq 32 \%$ (corresponding to a radius of $34^{\circ}$ for a single circular spot passing at the disk center) is found for a temperature ratio of about 0.84 . Given a maximum temperature $T_{\mathrm{ph}}=4666 \mathrm{~K}$, we obtain a spot temperature $T_{\mathrm{sp}}=3920 \mathrm{~K}$.

The solutions in the plane $\frac{T_{\mathrm{sp}}}{T_{\mathrm{ph}}}-A_{\text {rel }}$ for HK Lac are shown in Fig. 16. The minimum projected fractional area $34 \%$ (corresponding to a radius of $35^{\circ}$ for a single circular spot passing at the disk center) is obtained for a temperature ratio of about 0.83 . The assumed temperature maximum is $T_{\mathrm{ph}}=$ $4765 \mathrm{~K}$, and the corresponding spot temperature is $T_{\mathrm{sp}}=$ $3955 \mathrm{~K}$. 


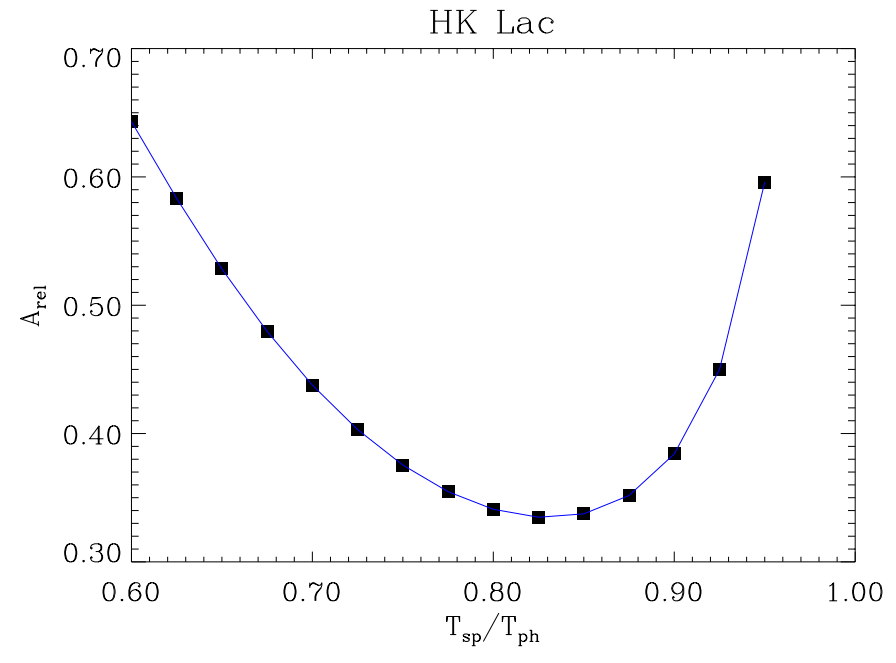

Fig. 16. Solutions of HK Lac $T_{\text {eff }}$ curve for different spots temperature $\frac{T_{\mathrm{sp}}}{T_{\mathrm{ph}}}$. A lower limit for the fractional area $A_{\text {rel }}$ of about $34 \%$ for $\frac{T_{\mathrm{sp}}}{T_{\mathrm{ph}}} \simeq$ 0.83 is apparent.

If the maximum temperature does not really represent the unspotted photospheric temperature, we would be underestimating the spots area at each fixed $\frac{T_{\mathrm{sp}}}{T_{\mathrm{ph}}}$. In any case, we are considering the area of unevenly distributed spots, i.e. those giving rise to the observed modulation. We cannot argue, on the basis of only one temperature maximum value, the presence of a contribution from additional evenly distributed spot groups, like for example an equatorial spot belt or a large polar spot, because we would have information about the "unspotted temperature"; likewise the unspotted magnitude is needed for photometric analysis. The effect of such structures on average temperature is only to lower the presumed unspotted temperature by a few tens of degrees, but its influence over the solutions is very limited, because the observed relative variations $\frac{\Delta T_{\text {eff }}}{T_{\text {eff }}}$ are only of a few percent.

\section{Conclusion}

The line-depth ratio method has proved to be very effective in detecting low variations of the disk-averaged temperature in active stars. The simultaneous use of many line pairs remarkably enhances the precision of the temperature measurements. Though the precision of the absolute value of temperature is limited by the accuracy with which the temperature scale itself can be established, i.e. $\sim 100 \mathrm{~K}$ (e.g. Gray 1992), the resolution in temperature is much higher, with errors in the $5-15 \mathrm{~K}$ range.

A well-defined rotational modulation of the average temperature, with amplitudes ranging from $119 \mathrm{~K}$ to $177 \mathrm{~K}$, has been detected in all three systems studied in the present work.

Though an accurate modelling of the temperature curves could give information on spot distribution, in the present work we have performed a simple analysis of these curves, based essentially on their variation amplitude interpreted in terms of a dark spot crossing the star disk during its rotation. We have derived the possible solutions for such a spot in the parameters space $\frac{T_{\mathrm{sp}}}{T_{\mathrm{ph}}}-A_{\text {rel }}$ (fractional temperature, fractional area). The possible solutions define a parabolic-shaped locus in the plane $\frac{T_{\mathrm{sp}}}{T_{\mathrm{ph}}}-A_{\text {rel }}$, providing a lower limit for the fractional area of the starspot (or starspots). The lower limits we found indicate large spot coverage in all stars, with relative areas of $41 \%$, $32 \%$ and $34 \%$ of star disk for VY Ari, IM Peg, and HK Lac, respectively. The filling factors, in units of star surface, are of $11.7 \%, 8.5 \%$, and $9.0 \%$, respectively. The temperature difference between spot and photosphere corresponding to these filling factors is $\Delta T \simeq 890 \mathrm{~K}$ for VY Ari, $\Delta T \simeq 750 \mathrm{~K}$ for IM Peg, and $\Delta T \simeq 810 \mathrm{~K}$ for $\mathrm{HK} \mathrm{Lac}$.

Values of $\Delta T$, obtained by means of light and color curve analysis, comparable with ours have been found by previous investigations into these systems.

For instance, Eaton \& Poe (1986) found $\Delta T \simeq 760 \mathrm{~K}$ for VY Ari from a spot modelling applied to their $B V R I$, while Strassmeier \& Bopp (1992) found $\Delta T \simeq 1200 \mathrm{~K}$ from their $U B V R I$ photometry.

For IM Peg, values of $\Delta T \simeq 920 \mathrm{~K}$ (Poe \& Eaton 1985) and $\Delta T \simeq 1130 \mathrm{~K}$ (Padmakar \& Pandey 1999) are reported.

For HK Lac, values of $\Delta T \simeq 950 \mathrm{~K}(\operatorname{Vogt} 1981), \Delta T \simeq$ $1080 \mathrm{~K}$ (Poe \& Eaton 1985), and $\Delta T \simeq 1200 \mathrm{~K}$ (Olàh et al. 1997) are reported.

We remark that, although the spot parameters we find are only indicative, because they are based only on a lower limit of the spot filling factor, they are well within the values found with other methods.

The simultaneous use of LDR and photometry, combined with a detailed spot-model, can lead to univocal solutions, since we expect a different behaviour of the locus of light-curve solutions in the $\frac{T_{\mathrm{sp}}}{T_{\mathrm{ph}}}-A_{\text {rel }}$ plane that can solve the ambiguity.

This matter will be the subject of a forthcoming paper.

Acknowledgements. This work has been supported by the Italian Ministero dell'Istruzione, Università e Ricerca (MIUR) and by the Regione Sicilia which are gratefully acknowledged. We are grateful to prof. D. Gray for several useful suggestions. We would like to thank the referee, Dr. Artie Hatzes, for his helpful comments and suggestions.

\section{References}

Bashkin, S., \& Stoner, J. O. Jr. 1975, Atomic Energy Levels \& Grotrian Diagrams (North-Holland Publ. Comp. - Amsterdam, Oxford Am. Elsevier Publ. Comp. Inc. - New York)

Berdyugina, S. V., Berdyugin, A. V., Ilyin, I., \& Tuominen, I. 2000, A\&A, 360, 272

Eaton, J. A., \& Poe, C. H. 1986, IBVS, No. 2846

ESA 1997, The Hipparcos and Tycho Catalogue, ESA SP-1200

Frasca, A., Marilli, E., \& Catalano, S. 1998, A\&A, 333, 205

Gorza, W. L., \& Heard, J. F. 1971, Publ. David Dunlap Obs., 3, 107

Gray, D. F. 1989, ApJ, 347, 1021

Gray, D. F. 1992, The Observation and Analysis of Stellar Photospheres, 2nd ed. (Cambridge University Press), 344

Gray, D. F. 1994, PASP, 106, 1248

Gray, D. F. 1996, in Stellar Surface Structure, ed. K. G. Strassmeier, \& J. L. Linsky (Dordrecht: Kluwer), IAU Symp., 176, 227

Gray, D. F., \& Baliunas, S. L. 1995, ApJ, 441, 436

Gray, D. F., Baliunas, S. L., Lockwood, G. W., \& Skiff, B. A. 1992, ApJ, 400, 681

Gray, D. F., Baliunas, S. L., Lockwood, G. W., \& Skiff, B. A. 1996a, ApJ, 456, 365 
Gray, D. F., Baliunas, S. L., Lockwood, G. W., \& Skiff, B. A. 1996b, ApJ, 465, 945

Gray, D. F., \& Brown, K. 2001, PASP, 113, 723

Gray, D. F., \& Johanson, H. L. 1991, PASP, 103, 439

Gray, D. F., \& Livingston, W. C. 1997, ApJ, 474, 802

Hatzes, A. P., Cochran, W. D., \& Bakker, E. J. 1998, ApJ, 508, 380

Henry, G. W., Eaton, J. A., Hamer, J., \& Hall, D. S. 1995, ApJS, 97, 513

Henry, G. W., Fekel, F. C., Henry, S. M., \& Hall, D. S. 2000, ApJS, 130, 201

Hoffleit, D., \& Warren, W. H. Jr. 1991, Bright Star Catalogue, 5th revised ed. (preliminary version), http://cdsweb.u-strasbg.fr/cats/Cats.htx

Huenemoerder, D. P., \& Ramsey, L. W. 1987, ApJ, 319, 392

Mermilliod, J. C., Mermilliod, M., \& Hauck, B. 1997, A\&AS, 124, 349

Moore, C. E., Minnaert, M. G. J., \& Houtgast, J. 1966, The Solar Spectrum $2935 \AA$ to $8770 \AA$ (National Bureau of Standards: Washington), Monograph 61

Neff, J. E., O’Neal, D., \& Saar, S. H. 1995, ApJ, 452, 879

Oláh, K., Kövári, Zs., Bartus, J., et al. 1997, A\&A, 321, 811
O’Neal, D., Saar, S. H., \& Neff, J. E. 1996, ApJ, 463, 766

Padmakar, \& Pandey, S. K. 1999, A\&AS, 138, 203

Piskunov, N. E. 1991, in The Sun and Cool Stars, Activity, Magnetism, Dynamos., ed. I. Tuominen, D. Moss, \& G. Rüdiger (SpringerVerlag, Berlin), IAU Coll., 130, 309

Poe, C. H., \& Eaton, J. A. 1985, ApJ, 289, 644

Ramsey, L. W., \& Nations, H. L. 1980, ApJ, 239, 121

Rice, J. B., \& Strassmeier, K. G. 2000, A\&AS, 147, 151

Strassmeier, K. G., Bartus, J., Cutispoto, G., \& Rodonò, M. 1997, A\&AS, 125, 11

Strassmeier, K. G., \& Bopp, B. W. 1992, A\&A, 259, 183

Strassmeier, K. G., \& Fekel, F. C. 1990, A\&A, 230, 389

Strassmeier, K. G., Hall, D. S., Fekel, F. C., \& Scheck, M. 1993, A\&AS, 100, 173

Strassmeier, K. G., Hall, D. S., \& Henry, G. W. 1994, A\&A, 282, 535 Strassmeier, K. G., \& Schordan, P. 2000, Astronomische Nachrichten, 321,277

Toner, C. G., \& Gray, D. F. 1988, ApJ, 334, 1008

Vogt, S. S. 1979, PASP, 91, 616

Vogt, S. S. 1981, ApJ, 150, 327

Vogt, S. S., Penrod, G. D., \& Hatzes, A. P. 1987, ApJ, 321, 496 